\title{
Teilhard de Chardin y su "frente humano"
}

Tras lo expuesto en los capítulos precedentes ${ }^{1}$, aparece claro el sentido de la crisis actual de la humanidad ante un nuevo umbral de crecimiento, así como la irresistible planetización que nos espera. Junto a las fuerzas coercitivas de la doble compresión geográfica y mental, y en estrecha colaboración con la energética amorizante del Punto Omega, he dejado indicado el decisivo papel que juegan la libertad y el esfuerzo humano en la orientación concreta que ha de tomar la totalización humana: colectivismo o personalismo comunitario.

Se hace urgente, por tanto, una toma de conciencia generalizada de nuestra situación y de la responsabilidad cósmica que nos atañe. Porque, en definitiva, será nuestra opción y nuestro esfuerzo quienes decidirán el éxito de la evolución en un universo personalizado o su abortamiento, al menos parcial, en un colectivismo mecanizante o un pluralismo regido por las fuerzas del egocentrismo.

Pero no se trata sólo de un problema de mentalidad, sino que, fundamentalmente, es un problema de acción. Teilhard de Chardin

1 Este trabajo consitituye uno de los capítulos - con ligeras variantes- de una tesis doctoral inédita, La antropología educativa de Teithard de Chardin. Téngase en cuenta esta advertencia para comprender determinadas alusiones, ya que a veces se apunta solamente lo que se ha estudiado antes o se estudiará más tarde. Utilizo las siguientes siglas:

Ecrits, por Ecrits du temps de la guerre (1916-1919). Grasset, 1965.

ETG, por Escritos del tiempo de la guerra (1916-1919). Taurus, 1967.

LdV, por Lettres de voyage (1823-1955). Grasset, 1956.

CV, por Cartas de viaje. Taurus, 1966.

NCV, por Nuevas cartas de viaje. Taurus, 1967.

AllH, por Accomplir l'Homme. Letitres inédites (1926-1952). Grasset, 1968.

LLZ, por Lettres à L. Zanta. Desclée de Brouwer, 1965 .

GdiP, por Génèse d'une pensée. Lettres (1914-1919). Grasset, 1961.

VII, por L'Activation de l'énergie. Seuil, 1963.

$\mathrm{AE}$, por La activación de la energía. Taurus, 2. ${ }^{\mathrm{a}}$ ed., 1967.

VI, por L'Energie humaine. Seuil, 1962.

$\mathrm{EH}$, por La energía humana. Taurus, 2. ${ }^{\mathrm{a}}$ ed., 1967.

$\mathrm{V}$, por L'Avenir de l'Homme. Seuil, 1959.

PH, por El porvenir del hombre. Taurus, $4 .^{\mathrm{a}}$ ed., 1967.

III, por La vision du passé. Seuil, 1957.

IV, por Le Milien divin. Seuil, 1957. 
insiste en este aspecto. Es preciso formular objetivos precisos y conseguir una organización eficiente, que orienten y canalicen el esfuerzo humano - la «energética humana»- al fin deseado. Es lo que Teilhard perseguía con su «frente humano». Las fuerzas educativas tienen una función transcendental —ontogenética y filéticaque cumplir. Pero, en su contexto cósmico, la teoría educativa adquiere una dimensión nueva: antropogenética, universalista y comunitaria:

«He aquí la condición del Progreso bumano, tal como la guerra nos la mostrado: que los hombres, cesen por fin de vivir aisladamente y lleguen a percibir un objetivo común para sus vidas (un Fin fijado desde siempre en su cielo, transmisible por la educación, alcanzable y perfectible por la investigación), bacia el que se enciendan y se agrupen (en un esfuerzo no individual ni regional, ni social, sino bumano) las potencias que abrigan todavía, seguramente, en ellos. Este fin común y superior, este Ideal esperado, cuya atracción tiene que acercarnos y perfeccionarnos, existe [...]: toda bambre tiene en alguna parte un pan que la espera» 2.

Porque, a nueva situación son exigitivas nuevas soluciones: «Una cosa es segura: vamos hacia nuevas situaciones, que exigirán soluciones y mentalidades nuevas» ${ }^{3}$.

\section{a) ESFUERZO HUMANO Y RESPONSABILIDAD COMUNITARIA.}

Nuestra generación y las siguientes deben enfrentarse con «una nueva cuestión de Galileo», de vital trascendencia, una vez descubierto el proceso «rápidamente acelerado de totalización humana», su sentido y sus condiciones. «Situados por la Vida en esta situación crítica, ¿cómo vamos a reaccionar frente a esta prueba?». Porque es preciso «tomar posición y poner manos a la obra, de prisa, inmediatamente». No podemos olvidar que estamos en régimen de autoevolución. Se precisa, pues, «un gesto, apasionada y conscientemente deseado». No basta una reacción «instintiva y pasiva», sino «una colaboración activa e inmediata, un impulso vigoroso, a base de convicción y de esperanza. Porque la Evolución no espera» ${ }^{4}$.

Nuestra responsabilidad sobrepasa el ámbito meramente jurí-

2 Ecrits, 394 [ETG, 411-412]. Los primeros esbozos del "frente humano" se aprecian ya en la correspondencia recogida en G.dP, 345'-354.

3 Lettres de voyaqe, 243 「NICV. 337. La misma idea so repite insistentemente en su correspondencia. Cfr. AlH, 36; ib., 41; ib., 72; ib., 97-97; ib., 196.

4 VII, 303-305 [AE, 271-3]. 
dico y adquiere dimensiones orgánicas. Igualmente trasciende su sentido individualista para convertise en «solidaridad cósmica», dentro de «la interacción activa de las libertades». Ante el proceso de compresión planetaria se hace especialmente dramática, ya que nos hacemos responsables del signo colectivista o comunitario que adoptará. Se exige, por tanto, imperiosamente su «ascenso en extensión, en profundidad y en volumen». Sin olvidar que «con lo Jurídico, siempre se puede, con algún compromiso, llegar a un entendimiento, mientras que lo Orgánico, si se le viola, no perdona» ${ }^{5}$. Porque, además, el esfuerzo humano tiene una función propia e intrasferible que cumplir: es condición - provisoria, pero necesaria - del éxito evolutivo de la superhumanización:

«Fíjese bien en lo siguiente: no atribuyo ningún valor definitivo y absoluto a las diversas elaboraciones humanas. Creo que desaparecerán, fundidas en una totalidad nuevra e inimaginable. Pero admito que desempeñan una función provisoriamente esencial; que son fases insustituíbles e inevitables por las que debemos pasar (nosotros o la raza) en el transcurso de nuestra metamorfosis. No me gustan sus formias particulares, sino su función, que es la de construir. misteriosamente; primero, lo divinizable, después - por gracia de Cristo al posarse sobre nuestro esfuerzo- lo divino» 6 .

En efecto, existe un «esfuerzo necesario, sin el cual una cierta porción de ser no se realizará jamás» ${ }^{7}$. Y es, igualmente, una obligación antropogenética ${ }^{8}$. Pero este esfuerzo es doblemente humanizador cuando se realiza unificado, en equipo. Lo que también es una necesidad, ya que «el hombre aislado, ni piensa ni progresa» ${ }^{9}$.

Pero se trata de un esfuerzo y una responsabilidad comunita-

5 Iib., 211-221 [AE, 193-201].

6 Carta del 12 de dic. de 1919 (citada por C. Cuenot, P. Teilhard de Chardin. Taurus, Madrid, 1967, p. 561).

7 "C'est une de mes acquisitions de cette année que la conviction de l'effort nécessaire, de l'effort sans lequel une certaine portion d'être ne se réalira pas". GdP, 202-3 (28 dic. 1916).

8 "Grandir et se réaliser le plus possible, telle est la loi immanente à l'être. En nous ouvrant des aperçus sur une Vie iplus divine, je ne puis croire que Dieu nous ait dispensé de poursuivre, même dans son plan naturel, l'oeuvre de la Creation. Il me semble que ce serait "le tenter" que de laisser le Monde aller son train, sans essayer de mieux le dominer et de mieux le comprendre. Il faut s'efforcer de diminuer la mort et la souffrance [...]". GdP, 161-162 (8 sept. 1916).

"Le plus grand sacrifice que nous puissions faire, la plus grande victoire que nous puissions remporter sur nous-mêmes, c'est de surmonter l'inertie, la tendaner au moindre effort". Ib., 71 (1915).

9 "L'homme isolé ne pense pilus et ne progresse plus" (25 de mayo de 1923), $\mathrm{LdV}, 35[\mathrm{CV}, 43]$. Cfr. tb. GdP, 235. 
rios. Hay que fomentar, pues, simultáneamente, «el sentido de la Especie», «el espíritu de la Tierra». Teilhard declara su propósito de ser - y de hacer- «ciudadanos de la Tierra» por encima de cualquier nacionalismo o confesión religiosa: «El bombre verdadero es el que reúne o reunirá en sí la conciencia de toda la capa humana total». Es el mismo «Humano soy y todo lo humano me concierne» puesto en clave evolutiva, antropogenética ${ }^{10}$.

Al mismo tiempo descubrimos que la única ciencia, la única investigación realmente decisiva, es la «construcción del futuro» ${ }^{11}$. Para ello se hace preciso «un nuevo Palomar» para estudiar y pronosticar la dirección y las condiciones de la superhumanización ${ }^{12}$.

Porque lo decisivo es que este movimiento hacia el porvenir «pasa actualmente a través de nosotros en su forma más central y más viva. Representamos el frente actual de la onda». Nos hallamos, pues, ante una tarea de proporciones gigantescas, cargada de la máxima responsabilidad:

"¿Qué haremos nosotros, nosotros que somos los elementos conscientes, para favorecer su avance? ¿Qué organizaciones escogeremos? ¿Qué relaciones anudaremos entre los pueblos? ¿Qué vías abriremos? ¿Qué moral adoptaremos? ¿Hacia qué ideal agruparemos nuestras energías? ¿Con qué esperanza mantendremos en el corazón de la masa humana el sagrado gusto por la investigación y por el progreso?» 13 .

En efecto, «no se trata sólo de conocer; hay que hacer que, en nosotros, la Evolución avance más» ${ }^{14}$. La frase parece un eco de la tesis marxiana, pero tiene su inspiración propia, porque en Teilhard el pensamiento adquiere una dimensión cósmica y evolutiva ${ }^{15}$.

10 También ésta es una tesis frecuente en ensayos y correspondencia. Aunque, como geólogo y paleontólogo, su profesión le llevaba al estudio del pasado, Teilhard se orientó pronto hacia el futuro. Tenemos testimonios ya en 1923 (cfr. LdV, 41; ib., 50-51; ib., 59-60; ib. 61; ib., 64 [CV, 50-51, 61, 71, 72, 76]. Sin embargo, fue en 1935 cuando tomó plena conciencia de ello (ib., 186; ib., 187-8 [CV, 189-190; 192] ; AlH, 109; ib., 117; cffr. tib. el ensayo La décou verte du Passé (1935), III, 257-270 [VP, 227-237]. No obstante, el pasado constituye la base para la prospección del futuro (cfr. La Visión du passé (1949), III, 333-344 [VP, 293-302].

11 VII, 306-9 [AE, 274-7]. Cfr. nota precedente.

12 III, 255-6 [VP, 225].

13 Ib., 256 [VP, 225].

14. Ib., 254 [VP, 224].

15 Es una idea y una consigna frecuentemente repetida en sus ensayos y en su correspondencia: "Je voudrais exprimer ce qui pense un homme qui 
b) El «Espíritu de LA TIERRA».

Ya en 1926 proyectaba Teilhard escribir «el libro de la Tierra», en el que se proponía «canalizar» todas las energías humanas, actualmente tan difusas, hacia la «unión universal» ${ }^{16}$. Este proyecto no se realizó hasta 1931 con el ensayo L'Esprit de la Terre ${ }^{17}$, en el que presentaba las líneas de su cosmovisión en torno a la tesis central sobre el origen y la estructura «terrestre» del hombre y su destino trascendente dentro de esta misma inmanencia ${ }^{18}$. Diez años más tarde, Teilhard confirmaba y completaba estas perspectivas en otro ensayo cosmovisional, L'Atomisme de l'Esprit ${ }^{19}$.

El «espíritu de la Tierra» es el encargado de «continuar el movimiento» para llevar a feliz término la plena hominización. Es todo «el problema cósmico de la Acción». Se trata de promover eficazmente un movimiento a escala terrestre, plantetaria. De hecho, esta es la dirección que se insinúa cada vez más. Ninguno de nosotros, aisladamente, «llega a bastarse materialmente a sí mismo». Cada vez con más fuerza se establece «una vida bumana general». La humanidad adopta la forma de «un organismo reflexivo com-

ayant percé les cloissons et les plafonds des petits pays, des petites coteries, des petites sectes, émerge au-dessus de toutes ces catégories et se découvre enfant et citoyen de la Terre. Rien que la Terre, a dit Paul Morand dans un dernier bouquin. Les quatre mo's valent mieux que tout son livre". AlH, 56; ib. 58-59; ib., 65; LdV, 97; ib., 103-4 [CV, 105-6; 112-3]. Todos los textos citados corresponden a 1926-1927.

16 "Dans le livre de la Terre je voudrais faire réserver un paragraphe à la "puissance d'aimer" áparpillée, diffuse, actuellement dans la poussière humaine. On se préoccupe des réserves d'énergie mécanique sur la Terre. Quand dionc songera-t-on que les milliards de HP et de Kilowats ne sont rien, psychiquement, auprès des tensions et dies possibilités de transformation incluses dans nos esprits et nos coeurs? Quand donc cessera-t-on d'abandonner à des casuistes étroits, à des morales fixistes ou à des médecins freudistes, quand donc regardera-t-on autrement que comme une occupation de rêveurs mystiques le soin de sauver et de libérer ce qui est la sève essentielle du monde? Ce qui fait la puissance immortelle du christianisme et la source profonde des suciès de S. Paul c'est de canaliser vers une issue précise, ou du moins le long d'un axe précise, les espérances d'union universelle qui sont, en froid analyse psychologique, notre seule raison défnitive de faire la moindre action". AlH, 66 (28 nov. 1926); cfir. Lettres die voyage, 148-9 [CV, 154-5] (acababa de escribir L'Esprit de la Terre); cfr. tib. VI, 25 [EH, 21], nota.

17 VI, 23-57 [EH, 21-51]. En carta a su prima Margarita, Teilhard ponía en relación su opúsculo con la obra de J. JEANS, The Universe around us, en la que encontraba "una actitud absolutamente contraria a la mía", aunque "se ven al desnudo sus flaquezas [incomprensión absoluta del fenómeno vitail], que falsea la perspectiva". También lo relaciona con la autobiografía de Hu-Shih, con su "credo" infantil y materialista. LdV, 148-9 [15'4-5].

18 VI, 23-35 [21-31].

19 VII, 27-64 [AE, 25-62]. 
plejo». «En lo sucesivo, y menos que nunca, el hombre podrá ya pensar solo». En todos los campos, las investigaciones descansan, «de hecho, sobre una bóveda de pensamientos dispuestos en arbotante». No se trata de una simple suma, sino de «una sintesis» ${ }^{20}$.

En realidad, es el mismo «sentido de la Especie» que ha inspirado la evolución hasta el hombre. En la humanidad, tras la «curva de moleculización» que produjo la «pluralidad de conciencias reflejas», comienza a dibujarse su figura por encima de la actual «repulsión incoercible» hacia la constitución del «estado de personalidad» que le es propio.

Es cierto, hasta ahora «las afinidades» profundas de base se encuentran «todavía adormecidas». Pero la convergencia psíquica y la organización internacional la favorecen. Llega el momento en que «es necesario para el Hombre creer en la Humanidad más que en sí mismo, so pena de desesperar». Por encima de la nimiedad de nuestras diferencias infantiles, se alza «el sentido de la Tierra». $Y$ es que «el Mundo continúa: habrá un Espíritu de la Tierra» que nos conducirá a un «estado de unanimidad» ${ }^{21}$.

Este «sentido de la Tierra» nace del «sentido apasionado del destino común que arrastra, cada vez más lejos, a la fracción pensante de la Vida». El sentimiento de ser «ciudadano de la Tierra» emerge más allá de los círculos restringidos de la familia, patria y raza para descubrir «que la única Unidad humana verdaderamente natural y real es el Espíritu de la Tierra». La humanidad atraviesa ahora «la crisis de pubertad» que la llevará a su estado adulto, capacitándola para «controlar, despertar u ordenar las fuerzas emancipadas del Amor, las fuerzas dormidas de la Unidad humana, las fuerzas vacilantes de la Investigación... ${ }^{22}$.

\footnotetext{
20 Ib., 41-45 [AE, 38-42]; VI, 36 [EH, 31-2].

21 VI, 37-39 [EH, 32-4]; VII, 46-7 [A.E,43-4]. Teilhard saludó emocionadamente el anuncio del "Año Geofísico internacional", en 1954, como el "Año 1 de la Noosfera". LdV, 360 [NCV, 180-1].

${ }_{22}$ VI, 39-40 [EH, 34-5]. Al reflexionar sobre "la repercusión espiritual de la bomba atómica", Teillhard subraya las palabras del semanario norteamericano "The New Yorker": "Sólo una energía polílica dirigida hacia la realización de una estructura universal puede equilibrar la aparición en el mundo de las fuerzas atómicas". En efecto, de la explosión se deducen las siguientes consecuencias: $1 .{ }^{\mathrm{a}}$, un nuevo sentimiento de poder; $2 .^{\mathrm{a}}$, de un poder indefinidamente desarrollable; $3 .^{\mathrm{a}}$, el impulso de crecer y desarrollarse biológicamente; 4. ${ }^{\text {a }}$, un acrecentamiento del sentido de evolución (frente al "taedium vitae" coexistente). Todo ello nos impone una "opción de fondo" sobre el sentido que vamos a darle al nuevo "Espíritu de la Tíerra": un espíritu "prometeico o
} 
¡La Unidad humana! Nada parece tan utópico, tan imposible. Fuera de los lazos tan restringidos del amor sexual y familiar, los encuentros amistosos, el hombre muestra una «repulsión instintiva» hacia sus semejantes. En ocasiones parece preferir la compañía de los animales y «se eriza, de alguna manera, a la primera aparición de un hombre semejante a él mismo». Esto parece dar la razón a «los pluralistas». Pero se trata sólo de una primera impresión. Porque dada su estructura «inter-céntrica», debe existir una «atracción profunda», aunque «esta atracción duerme todavía, es cierto».

El hombre sigue comportándose como un niño egocéntrico en su vida ordinaria. Pero excepcionalmente se adivina la presencia del sentido humano en algunos signos. En esos casos excepcionales (desastre, combate, investigación) «iqué perfeccionamiento en sus potencias [...], qué plenitud cuando, en algunos momentos de peligro o entusiasmo, llega, en un destello, a las maravillas de un Alma común!». Estas iluminaciones ocasionales indican el inmenso potencial que puede despertarse con «una pasión común». Es «el amor de inter-unión, por encima del amor de atracción».

Queda también el gigantesco potencial de la investigación en equipo. Hasta ahora la investigación ha seguido la ley de «las necesidades individuales e inmediatas». A lo más, dentro de los estrechos marcos nacionales. Pero, «la edad de las naciones ha pasado. Se trata ahora para nosotros, si no queremos perecer, de sacudir los antiguos prejuicios y de construir la Tierra» ${ }^{23}$.

Cierto que no se trata de una empresa fácil: «la Tierra no tomará conciencia de sí misma más que a través de una crisis de Conversión». Aquí enlazamos una vez más con la postulada acción amorizante y personalizante de «un Centro de nuestros centros», que haga despertar «con sus rayos» un amor universal, «un enlace afectivo de dimensiones cósmicas», actuando como «un primer Motor hacia adelante» ${ }^{24}$.

faústico, espíritu de autonomía y de soledad" o "espíritu cristiano, espíritu de entrega y donación". Es decir, espíritu de "fuerza" o de "amor". Teilhard deja en suspenso el interrogante, pero adelanta que "sólo la segunda, pienso, se revela capaz, si reflexionamos, de conferir al universo en movimiento su plena coherencia espiritual [...]". V, 177-187 [PH, 173-183].

${ }_{23}$ VI, $40-46[\mathrm{EH}, 35-40]$. La última cita figura enteramente subrayada en el original. Sobre las "repulsiones instintivas". Teilhard confiesa ingenuamente sus propias dificultades: cfr. IV, 184-5 [MD, 159-160].

24 VI, 46-47 [EH, 42-51]; VII, 47-55 [44-53]. 
c) «EL FRENTE HUMANO»: EL PROYECTO.

La intensidad de reflexión que su experiencia directa en la guerra de 1914 le proporcionó, sirvió de catalizador no solamente del despertar de su genio, sino que le inspiró también la conciencia de una vocación especial que cumplir, al calor de las lecturas de Wells, Kipling y Benson. En efecto, en 1918 Teilhard habla de «mi evangelio» ${ }^{25}$, al mismo tiempo que desea una cátedra en el Instituto Católico de París como «plataforma de acción intelectual» ${ }^{26}$.

Esta conciencia de una misión especial que cumplir va concretándose paralelamente al desarrollo de su cosmovisión. La correspondencia es la mejor fuente para el estudio de esta evolución. Así, en 1923, confía a H. Breuil su deseo de entregar su vida a algo que «es superior a nosotros mismos» ${ }^{27}$. Tres años más tarde se lamenta de que sus ensayos no puedan publicarse, ya que siente la necesidad de crear un espíritu, una corriente de pasiones en el medio humano». En último término, sus investigaciones paleontológicas las estima en cuanto le pueden facilitar la difusión de su visión ${ }^{28}$.

En 1927 expresa «la necesidad que tenemos de un grupo de nuevos San Francisco, más amplios, agresivos y modernos que aquél en su manera de amar al Mundo, y también más lógicos y anticonvencionales en la práctica de su ideal». Sería iniciar una revolución realmente constructiva. Teilhard se muestra decidido a defender «salvajemente» su fe en la Humanidad «en toda ocasión y no importa contra quién» ${ }^{29}$.

Estos entusiasmos recibieron poco después un rudo golpe al verse definitivamente confinado en Extremo Oriente. Superada la dura crisis provocada por esta situación, Teilhard se rehace prontamente y reafirma más y más su postura. En julio de 1933 puede escribir: «No tengo más que una cosa que hacer: desarrollar más

25 GdP, 350-1. “Ce qui me calme c'est la confiance absolue que, si dans "mon évangile" il y a un vrai rayon de lumière, ce rayon luira, d'une façon ou d'une autre" (13 dic. 1918). Teilhard registra las coincidencias que le unen con Wells. Kipling y Benson en "la vogue de cet évangile nouveau (et bien ancien)". Ib., 354 .

26 Ib., 353 (1 de enero de 1919).

$27 \mathrm{LdV}, 63$ [CV, 75].

28 AlH, 55; ib., 69; ib., 82.

29 Ib.. 89; ib., 96-7. La idea de "un nuevo San Francisco" aparece ya en una carta de 1921 (citada por C. CUENOT, op. cit., 560, nota 47), 
intensamente que nunca lo que yo creo, y mezclarme más íntimamente que nunca en la sangre que yo sueño en corregir. Cuestión de amor más grande, no de revuelta. No sé si es posible. Pero quiero ensayarlo. Una cosa me parece clara: por una causa como esta, no tengo miedo de nada ${ }^{30}$.

Esta «causa» va tomando contornos cada vez más precisos: no sólo difundir un cristianismo renovado, sino establecer un «Frente Humano»:

«Lo que me impacienta es la necesidad que habría de obrar un reagrupamiento general de fuerzas, no sobre un «frente popular», sino sobre un «Frente Humano», con el programa siguiente: fe en el futuro, fe en el universalismo, fe en el primado de lo Personal... Habría que liberar y sintetizar un espíritu verdaderamente humano, en el que soñamos todos» ${ }^{31}$.

Cuando trata de «materializar» sus aspiraciones, Teilhard no encuentra mejor camino que activar «un renacimiento ( $¿$ una revolución?) cristiana». En efecto, el cristianismo es la única confesión religiosa capaz de «animar» un Universo-Personal ${ }^{32}$.

En las «febriles horas» que precedieron a la segunda guerra mundial, Teilhard confiaba a su corresponsal: «ya comprenderás que no me gusta demasiado esta deserción, pero espero mi hora (si es que ha de llegar alguna vez) y elaboro pacientemente mi «mensaje» (?) y mi plataforma. Me parece más importante crear una nueva idea de la actividad bumana, que embarcarse en la fiebre capciosa de un movimiento político que tiene ya sus jefes y hallará siempre su fieles. Pero no dejo de seguir anhelantemente las extrañas metamorfosis que padecemos sin comprenderlas demasiado» ${ }^{33}$.

Un año más tarde confirma estas perspectivas: «por lo que respecta a mi «evangelio», es claro que mis posibilidades y mis

30. Ib., 98-99. En 1931 había escrito finalmente L'Esprit de la Terre.

31 Iib., 112. El texto es de oct. de 1936. Cifr. tb. Lettres à L. Zanta (LLZanta), 127. Poco antes había citado las "fuerzas" que había que agrupar: "Fascisme, communisme, démocratie ne signifient plus rien. Je rêverais de voir le meilleur de l'Humanité se regrouper sur un esprit défini par les trois directions suivantes: Universalisme, Futurisme, Personnalisme, et se rallier au mouvement politique, économique qui se montrera techniquemant le plus capable de sauver ces tres conditions. Il y a vraiment quelque chose à dire là-dessus. Je le sens et je le sais". LdV, 206 [CV, 208]; cfr. tb. ib., 209 [CV. 211]. Por entonces redactaba Teilhard su ensayo Sauvons l'Humanité (1936), IX, 167-191 [CC, 153-175].

32 A.lH, 115. La carta está fechada el 15 de junio de 1937.

33 LdV (13 feb. 1938), 228 [CV, 221-2]. El subrayado es mío. 
aptitudes no me llevan a crear un movimiento social definido, sino a contribuir para realizar una especie de atmósfera espiritual, en la que cada vida en su totalidad reverberase, transformada, como una piedra preciosa por un rayo de luz: como si el universo entero se impregnase de un amor creador». Esencialmente, es el mensaje cristiano, pero enriquecido con la mejor savia de los panteísmos ${ }^{34}$.

El estallido de la guerra pudiera haber significado una dura prueba para su optimismo y su fe en el hombre. Desbaratados sus planes, Teilhard se vio forzosamente confinado en Pekín. Mal informado, suspendidas las actividades paleontológicas, Teilhard se centró en la redacción de nuevos ensayos sobre la planetización humana, una vez terminado $E l$ fenómeno bumano (1938-1940). Del movimiento fascista le molesta, sobre todo, su «anti-progresismo», actitud que descubre igualmente en los aliados ${ }^{35}$.

Fiel a su cosmovisión, Teilhard ve el único modo de superar los problemas que se ventilaban en el conflicto armado mediante «un ideal internacionalista». Sin embargo, sufre sin poder encontrar alguna misión concreta. Pero pronto comprende que lo único a su alcance es continuar escribiendo ${ }^{36}$. Le disgusta profundamente que los aliados piensen en un «reajuste» en lugar de una «renovación intelectual y moral» ${ }^{37}$.

Teilhard comprende que, a la terminación del conflicto, el cristianismo tendrá una prueba y una oportunidad: «lo que necesitamos no es una Restauración, sino un Renacimiento; no prudencia, sino una fe apasionada en algún futuro». De lo contrario, «lo que nos

34. AlH (26 felb. 1939), 154.

35 Ib., 148; ib., 166 y 171. De esta época son los ensayos L'Heure de choisir. Un sens possible de la guerre (1939); La grandie Option (1939); Les Unités humaines naturelles. Essai d'une biologie et d'une morale des races (1939); Reflexions sur le Progrès (1941); L'Atomisme de l'Esprit (1941); L'Esprit nouveau (1942); Universalisation et union: un effort pour voir clair (1942); Super-Humanité, super-Christ, super-Charité. De nouvelles directions pour l'avenir 1943).

36 AlH, 162; ib., 168

37 Ib., 171. "Depuis trois semaines la situation était assez claire; et depuis le commencement cette guerre me déplaisait parce que j'avais le sentiment (peut-être vous l'ai-je dit) que, du côté des Alliés il y n' y avait aucun esprit de conquête ni de renouveau: uniquement lutte pour préserver une conception bourgeoise et démodé de l'Homme. Il y a quelque chose d'afreusement primitif et "étroit dans la religion et l'idéal de Hitler. Mais les allemands avaient une flamme intérieure et c'est cela qui était trop fort nous, beaucoup plus que les tanks". I.b., 174. Cfr. tb. L. dle Voyage, 257-8 [VIC, 52]. 
espera para mañana es el materialismo marxista» ${ }^{88}$. En el fondo, no se trata de «un conflicto entre totalitarios y demócratas, sino entre movilistas y fijistas». Por eso, hay que superar el engaño de las denominaciones y los bandos para buscar «un progreso humano» con un grupo de elegidos «venidos de toda dirección, de toda clase y de toda confesión» ${ }^{39}$.

En 1941, «desde el tranquilo observatorio de Pekín», Teilhard siente que se acerca el momento en que

«la Humanidad habrá de dividirse (u optar) entre la fe o la falta de fe en un progreso espiritual de la Tierria. El frente bumano, de que hemos hablado tantísimas veces... Por esto me siento poderosamente decidido a constituirme, por todos los medios posibles, en defensor de la idea y de la realidad del Progreso (colectivo y personalizante) contra todos los pesimismos laicos y religiosos. Sobre este credo elemental han de agruparse, desde los cuatro ángulos del horizonte, los «soldados de Gedeón» ${ }^{40}$.

\section{«Al cabo de tanto años de reflexión», Teilhard se siente ple- namente seguro en su perspectiva:}

«Todo me confirma en la convicción de que el futuro no puede ser forzado ni llevado más que por el grupo de quienes se reúnan en una fe común en el futuro espiritual de la Tierra. "Atrás - me atrevería a decirlos pesimistas ateos y los cristianos pesimistas». Mejor sentada científicamente, y mejor concebida filosóficamente, hemos de volver a la idea (o si te gusta más), al «mito» del Progreso. En esta atmósfera esencial veo yo el renacimiento a la vez del Humanismo y del Cristianismo. Al cabo de tantos años de reflexión, me parece que me hallo científica, filosófica y religiosamente armado para atacar este punto tan duramente discutido y de tal manera estratégico, este punto que rige, como una bifurcación, todo el futuro de la Noosfera. A los sesenta años, me parece que, al fin, be descubierto o precisado mi auténtica vocación. El problema es hallar ahora la plataforma sobre que subirme, el gesto que deba hacerse. Ya sabes que, quizá equivocadamente, no me gusta forzar los acontecimientos. Que el Señor me ayude a no perder la ocasión, en cuanto se me presente» ${ }^{41}$.

\section{de 1940.}

38 L. de voyage, $260[\mathrm{NCV}, 55]$; ib., 264-6 [NCV, 60-62]. Las cantas son

39 "A mon avis, le monde de demain naîtra du grouppe "elu" de ceux (venus de toute direction, de toute classe, et de toute confession à travers le monde humain) qui décideront qu'il y a quelque chose de grand qui nous attend en avant et qui donneront leur vie pour l'at'eindre. Les gens doivent opter pour ou contre le progrès maintenant. Et ceux qui disent non, il n'y a qu'à les laisser derrière. Et ceux qui disent oui découvriront bientôt qu'ils parlent le même langage et que même ils adorent le même Dieu. Voilà ce que j'aimerais dire à New York. Et voilà la cause pour laquelle je suis décidé à lutiter, aussi longtemps que je vivrai, en tout cas". AlH (9 febr. 1941), 185-6; cfr. tb. ib. 188-9. Teilhard se refiere al Congreso Universal de Creyentes, que se celebró en Nueva York en 1942, al que pensaba asistir, aunque no le fue posible.

$40 \mathrm{LdV}, 268$ [NCV, 65] (11 enero 1941).

41 Ib., 269 [NCV, 66-67]. 
En 1945 está ya impaciente por actuar ${ }^{42}$. Pero hasta el año siguiente no puede llegar a París. La capital francesa es, entonces más que nunca, un hervidero de ideas y pasiones. Teilhard desarrolla una intensa actividad. Se pone en contacto con E. Mounier y su grupo personalista, pero no consigue entenderse con G. Marcel $\mathrm{y}$ «los humanistas». Inicia entonces su cordial amistad con Julian Huxley, entonces presidente de la Unesco. Recibe en 1947 la «Legión de Honor» y es elegido para la Academia de Ciencias ${ }^{43}$.

El año 1948 marca una fecha decisiva en la vida de Teilhard de Chardin. El, que se siente un «nuevo Galileo», se ve obligado a renunciar a la publicación de su obra y a la cátedra del Colegio de Francia, dos objetivos tan ardientemente deseados como «plataforma». Su visita a Roma sólo sirvió para comprobar, esta vez más de cerca, su total incomprensión respecto de su mensaje ${ }^{44}$. Además, se ve relegado nuevamente al extranjero, esta vez en Nueva York.

Superada una crisis de desarraigo, Teilhard reemprende con nuevos ánimos la difusión de su «frente humano». Proyecta por entonces escribir un «tratado de antropogénesis» ${ }^{45}$. En 1949 se refiere a «mis amigos del Frente bumano» y se sorprende de constatar cómo «poco a poco, el movimiento hacia una conciencia internacional crece regularmente bajo el tumulto político» ${ }^{46}$. Llega entonces a idear un test para medir el «índice humano-evolutivo» ${ }^{47}$.

Al mismo tiempo reflexiona y escribe incesantemente sobre lo ultrahumano colectivo ${ }^{48}$. Poco después ingresa en la Academia de Ciencias y continúa su intercambio de ideas con Julian Huxley y otros antropólogos y humanistas ${ }^{49}$. En 1951 fija su residencia definitivamente en Nueva York y colabora con la «Werner Gren Fun-

\footnotetext{
42 "J'ai vécu ici en eaux calmes; et ce n'est pas une très bonne chose pour un soi-disant "prophète". AlH, 198 (10 oct. 1945).

43 Como "correspondiente". La elección como académico ocurrió en 1950. Las distinciones le importan únicamente desde el punto de vista de la "plataforma" para difundir su mensaje: AllH, 213; ib., 237.

${ }_{44}$ Ib., 229-232; LdV, 296-7 [NCV, 101].

45 A.lH, 232. No llegó a redactarlo, aunque las ideas centrales de su proyecto pasaron a los opúsculos Le Groupe zoologique humain (1949), La structure phylétique du groupe humain (1951) y Les singularités de l'espèce humaine (1954).

46 Ib., 241 Aldous.

Lb., 151. Por este índice pasaba Julian Huxley, pero no su hermano

48 Ib., 234

49 LdV, 301-302 [NCV, 108-9].
} 
dation» en nuevas expediciones paleontológicas en Sudáfrica, a la vez que planea sus propios objetivos ${ }^{50}$.

Mientras, J. Huxley ha puesto en marcha su The bumanist Frame ${ }^{51}$ y J. Rueff prepara un simposio «sobre problemas humanos» en París, al que concurrían físicos y biólogos ${ }^{52}$. En 1954 participa en el simposio de la Universidad de Columbia, en el que colaboran científicos y humanistas. Estos últimos -Gilson, Battaglia, Malik- le decepcionan por su inmovilismo ${ }^{53}$. En el mismo año saluda con júbilo el anuncio de un «año geofísico internacional», al que denomina «año 1 de la Noosfera» ${ }^{54}$.

La perspectiva de un final cercano le entristece, ya que no ha tenido tiempo ni ocasión de «hacer circular» su pensamiento ${ }^{55}$. Una idea le obsesiona: «terminar bien», para que su mensaje no sufra detrimento ${ }^{56}$. No obstante, se preparaba a morir con una certeza:

50 "J'entrevois trois plans d'activité à la Foundation:

1. ${ }^{\circ}$ Travailler à l'organisation des recherches concernant l'Homme fossile (et les origines humaines).

2. Amorcer (c'est plus délicat) un type inédit de recherches orientiées vers la détection et vérification scientifiques de ce que j'apelle une "convergence" chez l'Homme de l'Evolution sur elle-même.

3. Poursuivre (de façon complètement privée) mon effort pour repenser la Christologie et le Christianisme en fonction d'une Humanité en voie de convergence biologique.

Comme tu le sais, c'est le deuxième point qui me paraît présentement décisif, en bonne strategie". Ib., 319-320 [NCV, 129-130].

51 Proyecto muy similar al de Teilhard, aunque sin un Punto Omega final. LdV, 234 [NCV, 148]. Cifr. J. HuXLEY, Essays of a Humanist. Londres, 1964, 76-119.

52 Ld' 340 [NCV, 156-7].

53 Sujet de Symposium: l'unité du savoir humain. Dans ma section, un profond - et vif- clivage s'est manifesté entre humanistes et scientistes, portant au fond, comme je l'ai dit en séance finale sur la nouvelle question de Gallilée: "L'Homme se meut-il encore biologiquemenit sur lui-même?". J'ai naturellement pris vigoureusement position (avec Huxley et la grande majorité des scientistes) contre la position immobiliste, hélas, des éléments les plus christianisés de la section, à savoir Gilson, Malik [...], Battaglia [...] et même Van Düssen". L. de voyage, 358 [NCV, 178]. En realidad, esta decepción arranca desde mucho antes. Teilhard ha insistido largamente en que la antropología debe convertirse en antropogénesis, rompiendo los viejos moldes del "humanismo filosófico". Cfr. C. Cuenot, TC, 489-506; AlH, 125.

54. LdV, 360 [NCV, 180-1].

55. En una carta de esta época confiaba a su gran amigo $\mathrm{H}$. Breuil: "Je comprendis que vous souhaitiez dix ans de plus et je prie le Seigneur de vous les donner. En toute hypothèse, vous avez déjà réussi à faire passer l'essentiel de votre vision et de votre oeuvre dans la circulation définitive de la pensée humaine. Et cette considération doit vous donner une grande paix". LdV, 363, nota 2 [NCV, 185, nota 29 ].

56 "Les ombres s'allongent (et se multiplient) autour de nous. Ma grande prière (je la fais aussi pour tous ceux que j'aime) est de "bien finir"; j'entend de "sceller", d'une façon ou de l'autre par ma mort, ce pour quoi j'aurai toujours vêcu". LdV', 363 (8 de ener ode 1955) [NCV, 185']. 
su movimiento se abriría paso, antes o después; «un día u otro, se establecerá la cadena», porque «basta que la Verdad aparezca una sola vez, en un solo espíritu, para que nada pueda ya jamás impedir que lo invada todo y lo inflame todo» ${ }^{57}$.

\section{d) «EL FRENTE HUMANO»: LAS BASES.}

\section{En abril o mayo de 1936 escribía Teilhard:}

«Me agobia la agitación humana del día (y muchos de mis amigos piensan como yo), y sufro al ver a tantos hombres volver a un conservadurismo tradicional bajo la presión de los hechos. Me parece, pues, que ha llegado el momento de tirar por la calle de en medio. Fascismo, comunismo, democracia, ya no significan absolutamente nada. Soñaría con ver agruparse lo mejor de la humanidad en torno a un espíritu definido por las tres coordenadas siguientes: Universalismo, Futurismo, Personalismo, y unido al movimiento político, económico, que técnicamente muestre ser el más capaz de salvaguardar esas tres condiciones. Hay mucho que decir sobre esto, en verdad. Yo lo sé y lo siento» 58 .

Pocos meses más tarde, Teilhard tiene listas «las bases» de este «frente humano» llamado a recoger y agrupar todas las fuerzas vivas, procedentes de toda afiliación política o confesional. Es el

Ya el 8 de nov. de 1953 habría escrito: "La seule chose claire étant que je voudrais employer aussi intensément que possible les dernières années que me restent à "christifier" (comme je dis) l'Evolution [...]. Cela, -et puis bien finir-, c'est-à-dire mourir en témoignage de cet "évangile". L.dV, 351 [NCV, 169]. La idca se repite insistentemente desde 1953 (cfr. LdV, 349-368 [NCV, $167-191]$

57 "Partout sur Terre, en ce moment, au sein de la nouvelle atmosphère spirituelle créé par l'aparition de l'idée d'Evolution, flottent, à un état de sensibilisation mutuelle extrême, l'amour de Dieu et la foi au Monde: les deux composantes essentielles de l'Ultra-Humain. Ces deux composantes sont partout "dans l'air": mais généralement pas assez fortes, toutes les deux à la fois, pour se combiner l'une avec l'autre, dans un même sujet. En moi, par pure chance (itempérament, éducation, milieu...), la proportion de l'une et de l'autre se trouvant favorable, la fusión s'est opéré spontanément - trop faible encore pour se propager explosivemen:- mais suffisante toutefois pour établir que la réaction est possible, et que, un jaur ou l'autre, la chan̂ne s'établirá.

Preuve nouvelle qu'il suffit, pour la Vérité, d'apparaître une seule fois, dans un seul esprit, pour que rien ne puisse, jamais plus, l'empêsher de tout envahir et de tout enflammer". Le Christique, 14-15 (1955). Es la conclusión del opúsculo.

Su prima Margarita aporia también el mismo testimonio: "Il ne s'attendait guère à être entendu dans l'immédiat, "mais l'idée une fois lancée peut faire son chemin, disait-il, it faut semer à tout vent". LdV, 367 [NCV, 190]. E. BoRNE ha escrito certeramente: "Tel a été le grénie du P. Teilhard qui, du savoir positif et de l'impatience prophétique a fait une indivisible grandeur" ("Un grand penseur religitux", Le Monde, 13 abril 1955).

58 LdV, 206 [CV, 208]. Los subrayados son míos Cfr. nota 31. 
ensayo Salvemos a la bumanidad. Teilhard se propone trazar unas directrices claras para este movimiento, que significan tanto «una profesión de fe» como una «concepción objetiva de los acontecimientos en curso» ${ }^{59}$.

Lo que bay que creer: el porvenir bumano.-Hay que levantarse sobre la inevitable miopía que provoca la excesiva cercanía de los acontecimientos, para buscar la perspectiva adecuada. De este modo se consigue un diagnóstico más adecuado. Por encima de los pesimismos actuales, hay que comprender que la humanidad atraviesa una crisis de crecimiento. El eje de complejidad-conciencia lo demuestra. «Avanzamos» y ante nosotros se nos abre un horizonte «ilimitado» e «irreversible» hacia nuestra suprema personalización. Es una situación «de derecho» que la acción humana debe convertir en actualidad «de hecho». De este modo comprobamos que «totalización y personalización son las dos expresiones de un único movimiento». Por tanto, conocemos la dirección: «Futurismo, Universalismo, Personalismo», son «los ejes inquebrantables» sobre los que debe apoyarse «el esfuerzo humano», «las tres columnas del porvenir» ${ }^{60}$.

Lo que bay que ver: la convergencia bumana.-Los movimientos actuales se reducen a «tres influencias fundamentales» que se sitúan «fuera del Cristianismo y luchan cada una de ellas por la posesión de la Tierra: Democracia, Comunismo, Fascismo». En ellas reconoce Teilhard «las tres aspiraciones que se nos manifestaban como características de la fe en el Porvenir. Pasión del Futuro, pasión de lo Universal, pasión de lo Personal». Ahora bien, la Democracia, originaria de la revolución, se ha diseminado en «falsos liberalismos intelectuales y sociales». $\mathrm{Ha}$ perdido su universalismo y ha confundido el individuo con la persona. El Comunismo, por su parte, ofrece una «auténtica seducción» al aunar su «evangelio humanitario» con «la fe en un organismo humano universal». Pero «la Materia ha velado el Espíritu» y amenaza «hacer del Hombre un termita». Finalmente, el Fascismo «está abierto al futuro», pero 
se apoya en «minorías selectas» y tiene una cosmovisión «de dimensiones neolíticas». De ahí que solamente el espíritu cristiano nos ofrezca las garantías suficientes con su abertura escatológica, su objetivo universal y su respeto a la persona ${ }^{61}$.

Lo que bay que hacer: el frente bumano.-Tras esta perspectiva, sólo se nos ofrece una actitud consecuente: «se trata de que ayudemos, con todas nuestras fuerzas, al nacimiento de un Mundo nuevo que aspira a salir a la superficie». Se trata de conseguir «una síntesis nueva» en la que se integrarían «el sentido democrático de los derechos de la Persona; la visión comunista de las potencias de la Materia; y el ideal fascista de las minorías selectas organizadas».

Nuestros antepasados hicieron causa común en la conquista «de la justicia y los derechos humanos». Nosotros perseguimos lo mismo, pero a escala de cosmogénesis, es decir, tratamos de conseguir «los Derecho del Mundo, en el nombre (no tan abstracto como parece) del Futuro, de lo Universal y de la Persona». Efectivamente, «en definitiva no hay más que dos grupos de Hombres en la Humanidad: los que arriesgan su alma en un Futuro más grande que ellos mismos, y los que, por inercia, egoísmo o desaliento, no quieren avanzar».

Naturalmente, no se trata de perseguir «una fórmula abstracta». Progresivamente irá surgiendo la organización adecuada: con los ingenieros de la Tierra surgirán los investigadores de «la Energética del Espíritu», lo que, finalmente, bien puede conducirnos «al descubrimiento de Dios» ${ }^{62}$.

El lugar del cristianismo.-El cristianismo ha sido la fe que ha inspirado las realizaciones humanas durante dos milenios. Ante un nuevo umbral de crecimiento en la humanidad, «está estrechamente comprometida su existencia». Pero observamos que, objetiva e imparcialmente, es la única fuerza capaz de inspirar el «frente humano», porque sus tres ideales constituyen cabalmente la médula de su mensaje de salvación. El cristianismo está llamado a convertise,

61 Ib., 178-183 [CC, 162-7]. Téngase en cuenta la fecha del ensayo: noviembre de 1936 .

62 Ib., 184-7 [167-171]. 
pues, en el alma del «frente humano», si es capaz de «re-encarnarse»: Si en lugar de buscar un objetivo «extra-terrestre», comprende que su objetivo es «supra-terrestre» ${ }^{63}$.

El pertinaz conflicto entre cristianismo y progreso, ciencia y religión, es solamente un problema de «transponer sus preceptos y sus consejos a las dimensiones de una Humanidad que se ha vuelto consciente de la inmensidad histórica y de las potencialidades o exigencias colectivas de su desarrollo». Sólo el cristianismo, entre las grandes religiones, es capaz de lograr esta transposición, ya que se adecua perfectamente con su potencial implícito. Por eso, le cabe al mensaje cristiano la función de «omegalizar» el frente humano mediante «toda una Filosofía de la Vida, toda una Etica, toda una Mística nuevas» ${ }^{64}$.

Filosofía de la vida.-El hombre necesita tener «un objetivo tangible para sus actividades». La propia dinámica personal exige un objetivo posible y probable para justificar el esfuerzo humano. ¿Por qué buscar, descubrir, construir siempre? El cristianismo nos ofrece «con Omega un objetivo y una atracción suprema para animar y dirigir el Esfuerzo humano». Además, nos presenta una solución satisfactoria de los tres grandes problemas: el mal, la desigualdad y la relación individual-social. El primero es «la pena de personalización». El segundo desaparece al comprender que cada elemento cumple su misión propia «en una masa convergente» hacia un mismo «éxito final». El tercero se soluciona al descubrir que «en un universo en vías de centración», el «individuo y la colectividad se refuerzan y se perfeccionan uno al otro, continuamente», hasta que, «en última síntesis», «el propio Todo se ha convertido en Persona» ${ }^{65}$.

Etica.-La máxima evangélica central: «amaos los unos a los otros» encierra, antes y siempre, el potencial básico de toda auténtica ética religiosa. La versión cristiana acentuaba, en un mundo en cosmos, «la caridad resignada y estática». Pero, en un mundo en cosmogénesis, se hace urgente realizar la transposición: «estamos

63 Ib., 187-191 [CC. 171-5].

64. VII, 55'-6 [AE, 53-4].

65 Iib., 56-8 [AE, 54-6]. 
cósmicamente destinados a convertirnos en uno». No bastan, por tanto, «las atenciones mutuas», sino que es preciso fomentar el progreso: «es «gasolina» y no únicamente aceite lo que necesitamos...». Ahora bien, la caridad cristiana «transpuesta a un Universo en vías de reagrupación espiritual, se dinamiza automáticamente» ${ }^{66}$.

Mística.- «La medida de una Etica es su capacidad para florecer en Mística». En efecto, «no hay Moral que viva sin rodearse de adoración». Pero se trata de una Mística activa: «Sentido bumano; más tarde, sentido de la Tierra; y por último, sentido de un Omega; tres etapas progresivas de una misma iluminación». Para la mística cristiana dinamizada, «cooperar significa incorporarse a una realidad viva. Obrar, en todas sus formas (con tal que sean positivas, es decir, unificadoras), equivale a comulgar». De este modo, todo se revaloriza, todo se hace susceptible de «omegalización». «En un universo reconocido como de naturaleza convergente, una neoespiritualidad para un neo-Espíritu» ${ }^{67}$.

e) Un nuevo Humanismo.

Constituídas las bases de la renovación que va a ser operada por el «frente humano», hay que plantearse el problema del «control y el mantenimiento» de la energía recién conquistada, es decir, el problema de su organización adecuada. Teilhard propone varios objetivos a realizar, dentro de lo que llama «un nuevo Humanismo».

Ante todo, moralizar la Invención.-Es decir, la actividad humana constructiva. Hasta ahora se ha regido «por un juego de necesidades externas, sobre todo económicas». Debido a ello estamos a punto de alcanzar la situación del «aprendiz de brujo». Y es que la técnica humana precisa también una «neo-mística», incluso en términos de «supervivencia». No podemos olvidar que, al situarse en régimen de auto-dirección evolutivo «se moraliza» automática-

66: Ib., 58-60 [AE, 56-8]. Sería preciso desarrollar estas proposiciones. Me propongo hacerlo en capítulos ulteriores.

67 Ib., 60-3 [AE, 59-62]. La última cita figura subrayada en su totalidad en el original. 
mente. De hecho, «allende cierto nivel, el progreso técnico va flanqueado necesariamente, funcionalmente, por el progreso moral». Ello prueba que «ambos movimientos son interdependientes» ${ }^{68}$.

Luego, alimentación espiritual del esfuerzo bumano.-Al hacerse reflexiva la evolución, se hace también crítica. La «energía vital» deja de ser una magnitud «fija en calidad y en cantidad». Con el hombre, la evolución, antes pasiva, tiende a hacerse activa al finalizarse». Por ello, «la energía creadora humana, según la temperatura interior a que sea elevada, es decir, entre el entusiasmo y la náusea, puede saltar en pocos instantes de más a menos el infinito».

Todo depende, pues, del grado de dinamismo que sepamos imprimir a nuestro esfuerzo creador. Condición previa indispensable es si el esfuerzo «vale la pena» o no. Ello equivale a saber si nos dirigimos a un objetivo irreversible y personal superior a nosotros. De aquí se desprende toda una energética educativa, el «coeficiente de activancia», es decir, «la aptitud más o menos grande que posee para excitar los centros de actividad refleja».

Hay que construir, pues, «una escala de activancias» según la capacidad respectiva para hacer «vivible» el Mundo, aunque, en realidad, «sólo un valor es capaz de satisfacernos: el mayor de todos». En todo caso, la construcción de esta escala presupone la elaboración de una «Noodinámica», esto es, la «dinámica de la energía espiritual» o «dinámica del espíritu», que se basa en «la necesidad de integrar el psiquismo humano, el Pensamiento, en una auténtica «Física» del Mundo» ${ }^{69}$.

Entre los objetivos inmediatos de solución que este «nuevo humanismo» debe satisfacer, se encuentran algunos de máxima urgencia, tales como «asegurar un eugenismo creciente, nutritiva, educativa y selectivamente». También debe fomentar al máximo «el desarrollo de las energías afectivas, generatrices últimas de la unión: sentido sexual sublimado y sentido humano generalizado». Ha de fomentar, igualmente, «el sentido de la Especie», «una fe colectiva (operante y unanimizante) en cierta maduración futura de la Huma-

$68 \mathrm{~V}, 259-262[\mathrm{PH}, 247-250]$.

69 Ib.. 262-267 [PH, 251-256]. Aquí se enlaza directamente la vertiente educativa de "la energética humana" y "la activación de la energía humana", que serán el objeto de estudio de los próximos capítulos. 
nidad». Teilhard llega a pedir la construcción de «un nuevo Palomar» para investigar con las máximas garantías científicas las condiciones de estudio y de energética requeridas para encauzar al máximo la acción humana, aunque añade que no se precisa tanto de «datos nuevos» como de «un nuevo modo de mirar y de aceptar los datos» ${ }^{70}$.

Bajo la perspectiva de este «nuevo humanismo», en efecto, nuestro Universo «se aclara a nuestra inteligencia», «se precisa ante nuestra libertad», «se valoriza en nuestra estimación», «se calienta para las potencias del amor» y se ilumina, es decir, se descubre capaz de satisfacer las más elevadas aspiraciones de nuestra mística» ${ }^{71}$. Ninguna garantía mejor podemos esperar de su validez objetiva.

Madrid, marzo 1970.

\section{José RuBio}

70 VII, 209-210 [AE, 190-2]; Ib., 306-9 [AE, 273-7]. Cfr. nota 69.

71 V, 118-122 [PH, 114-8]. En estas partes late toda una filosofía de la acción. 